\title{
Características físicas e sensoriais de Salame Tipo Italiano com adição de própolis
}

\author{
Physical and sensory characteristics of Italian-Type Salami with propolis addition \\ Daniele Cristina Savoldi ${ }^{1}$, Cléria Andréia Kunrath ${ }^{2}$, Débora Francielly de Oliveira ${ }^{3}$, Cláudio Roberto \\ Novello $^{2}$, Alexandre Rodrigo Coelho ${ }^{4}$, João Francisco Marchi ${ }^{2}$ e Ivane Benedetti Tonial ${ }^{\star}$ \\ ${ }^{1}$ Universidade Estadual de Londrina, Londrina, Paraná, Brasil. \\ ${ }^{2}$ Universidade Tecnológica Federal do Paraná, Francisco Beltrão, Paraná, Brasil. *Autor para correspondência: ivane@utfpr.edu.br. \\ ${ }^{3}$ Universidade Federal de Rondônia, Ariquemes, Rondônia, Brasil. \\ ${ }^{4}$ Universidade Tecnológica Federal do Paraná, Londrina, Paraná, Brasil.
}

\begin{abstract}
RESUMO
No presente estudo foram elaboradas quatro formulações de salame tipo italiano, sendo uma isenta de antioxidante (controle), uma adicionada de antioxidante sintético (BHT) e duas contendo extrato de própolis $(0,01 \%$ e $0,05 \%)$. As amostras foram submetidas a análises microbiológicas, sensoriais e perfil de textura e cor, além da perda de peso e o pH durante o período de maturação. Os resultados mostraram perda de peso das formulações durante a maturação, indicando que a incorporação da própolis não afetou o processo de secagem. A formulação adicionada de $0,01 \%$ de própolis apresentou coloração vermelha mais acentuada no final da maturação em comparação as demais. Os valores de $\mathrm{pH}$ variaram entre algumas amostras, bem como em relação ao dia de maturação. A adição de BHT acelerou o processo de desidratação do salame, deixando-o com maior grau de dureza. As formulações controle e aquela adicionada de $0,05 \%$ de própolis apresentaram melhores resultados para o perfil de textura, embora não tenham diferido das demais em relação ao atributo textura durante o teste sensorial. A formulação controle e a contendo BHT apresentaram os melhores resultados para intenção de compra e preferência. Por outro lado, todas as formulações apresentaram percentuais de certeza de compra acima de $70 \%$, sugerindo aceitação por parte dos consumidores.
\end{abstract}

PALAVRAS-CHAVE: antioxidantes, extrato de própolis, aceitação sensorial, características físicas, embutido cárneo.

\begin{abstract}
In this study, four formulations of Italian-type salami were prepared, being one antioxidant free (control), one containing synthetic antioxidant (BHT) and two containing propolis extract $(0.01 \%$ and $0.05 \%)$. The samples were submitted to microbiological and sensorial analyses and texture and color profiles, besides the weight loss and the $\mathrm{pH}$ during the maturation period. The results showed weight loss of the formulations during maturation, indicating that the incorporation of propolis did not affect the drying process. The formulation with $0.01 \%$ propolis showed a more intense red color at the end of maturation compared with the others. The $\mathrm{pH}$ values varied between some samples as well as in relation to the maturation day. The addition of BHT accelerated the dehydration process of salami at the end of the maturation period, making it harder. The control formulations and that with $0.05 \%$ propolis added showed better results for the texture profile, although they did not differ from the others in relation to the texture during the sensorial test. The control formulation and that containing BHT showed the best results for purchase intent and preference. Moreover, all formulations presented certainty percentage of purchase above $70 \%$, suggesting acceptance by consumers.
\end{abstract}

KEYWORDS: antioxidants, propolis extract, sensory acceptance, physical characteristics, meat sausage.

\section{INTRODUÇÃO}

O salame tipo italiano é um produto cárneo de elaboração tecnológica bem difundida, apresentando características sensoriais peculiares em termos de sabor, odor e textura (BELEDELLI et al. 2011). Segundo OLIVEIRA et al. (2013), a cor também apresenta influência na aquisição de um produto e por isso é também 
considerada um atributo de qualidade dos alimentos, principalmente quando se trata de industrializados cárneos, podendo interferir na aceitação do consumidor.

Estas características que representam a qualidade dos produtos cárneos podem sofrer alterações no período de armazenamento mediante reações de oxidação lipídica (OLIVO 2006), influenciados principalmente pelas condições de armazenamento e quantidade de oxigênio presente na embalagem (ZANARDI et al. 2002), sendo uma das maiores causas de deterioração, interferindo diretamente nos atributos sensoriais (ALCANTARA et al. 2012).

Retardar ou evitar o processo de oxidação lipídica é para a indústria cárnea um grande desafio, uma vez que se tem como propósito ofertar aos consumidores produtos com cor e sabor agradáveis, e que suas características de frescor e estabilidade se mantenham durante toda a sua vida de prateleira, com maior segurança e menor custo possível (MATHIAS et al. 2010).

Neste sentido, o uso de antioxidantes surge como alternativa para prevenir ou retardar a oxidação em alimentos gordurosos, como é o caso dos embutidos cárneos, de modo a evitar sabores e odores indesejáveis e manter a palatabilidade, aceitabilidade e valor nutricional dos mesmos (CAMPAGNOL 2007).

Os antioxidantes são compostos aromáticos, contendo pelo menos um grupo hidroxila, podendo ser sintéticos (butilhidroxianisol-BHA; butilhidroxitolueno-BHT), ou naturais (PEREIRA et al. 2010). De acordo com os mesmos pesquisadores, os antioxidantes naturais têm sido empregados em alimentos cárneos em substituição aos sintéticos uma vez que estes estão muitas vezes relacionados a efeitos adversos à saúde do consumidor. Assim, estudos direcionados a encontrar antioxidantes naturais que apresentem eficácia equivalente a um antioxidante sintético tem sido um grande desafio para indústrias e pesquisadores.

Dentre os compostos orgânicos que apresenta atividade antioxidante encontra-se a própolis, a qual é definida pela legislação brasileira como "o produto oriundo de substâncias resinosas, gomosas e balsâmicas, colhidas pelas abelhas, de brotos, flores e exsudatos de plantas, nas quais as abelhas acrescentam secreções salivares, ceras e pólen para elaboração final do produto" (MAPA 2001).

O extrato etanólico da própolis vem se destacando devido as suas propriedades farmacológicas, antimicrobianas, antiinflamatórias, cicatrizantes e antioxidantes, entre outras, vêm a proporcionar a sua aplicação pela indústria farmacêutica e indústria de alimentos bem como na produção de alimentos funcionais (ALVES \& KUBOTA 2013).

Além da função de conservantes, a utilização de antioxidantes em produtos cárneos fermentados pode influenciar no tempo de maturação e, consequentemente, no volume de perda de água do alimento ao término desse processo, requerendo, portanto, a aplicação de testes sensoriais para avaliação das suas características.

Considerando o exposto, o presente estudo teve por objetivo avaliar as características físicas e sensoriais de salames tipo italiano preparados com extrato alcoólico de própolis como antioxidante natural e outras duas preparações, uma com BHT e outra isenta de antioxidantes.

\section{MATERIAL E MÉTODOS}

\section{Obtenção e preparação da própolis}

Própolis in natura adquirida de produtores de mel do município de Francisco Beltrão, PR foi selecionada manualmente e triturada $(1,00 \mathrm{Kg})$, sendo posteriormente submetida a extração em etanol/água $70 \%(\mathrm{~m} / \mathrm{m})$ na proporção de $10 \%(\mathrm{~m} / \mathrm{m})$ de própolis em relação ao solvente. A mistura foi extraída por turbólise por 15 minutos de agitação sendo em seguida filtrada a vácuo (BRUSCHI 2006). Ao extrato obtido $(8,92 \mathrm{Kg})$ foram adicionados $125,36 \mathrm{~g}$ de HidróxiPropil Metil Celulose e $65,66 \mathrm{~g}$ de alginato de sódio (Protanal SF 120 RB- FMC Biopolymer), previamente dissolvidos em água. A mistura foi concentrada em rotaevaporador (Buchi-modelo R-153) e seca em Spray Drier (Labmaq-modelo MSD 1.0) onde se obteve $420 \mathrm{~g}$ de amostra.

\section{Elaboração do salame tipo italiano}

Foram desenvolvidas quatro formulações com 30 peças de $200 \mathrm{~g}$ cada, utilizando paleta, pernil e toucinho costo-lombar de suínos. A carne $\left(4^{\circ} \mathrm{C}\right)$ foi moída em disco de $8 \mathrm{~mm}$ e o toucinho picado em cubos $\left(0{ }^{\circ} \mathrm{C}\right)$ e homogeneizado para posterior adição dos demais ingredientes. Utilizaram-se como cultura starter (TEXEL ${ }^{\circledR}$ AS-308) as seguintes bactérias: Lactobacillus sakei, Staphylococcus carnosus, Staphylococcus xylosus e a dextrose como veículo. Após a adição dos ingredientes e aditivos, homogeneizou-se por mais 5 minutos. A Tabela 1 apresenta os ingredientes utilizados para elaboração dos salames tipo italiano.

O embutimento se deu em tripa artificial de colágeno $(25 \mathrm{~cm}$ de comprimento), em embutidora manual. Depois de 12 horas a $7 \stackrel{\circ}{\circ} \mathrm{C}$, os salames foram defumados com fumaça natural em umidade de $80 \%$ e temperatura de $30^{\circ} \mathrm{C}$ por 2 horas e 30 minutos. Na sequência, o produto permaneceu por um período de 
28 dias em câmara de maturação.

\section{Análises físicas e determinação de pH}

As análises físicas foram realizadas com amostras de salame escolhidas aleatoriamente. Todas as análises foram realizadas em duplicata para cada uma das três repetições de cada formulação, totalizando seis replicatas.

Tabela 1. Ingredientes utilizados em cada formulação do Salame Tipo Italiano.

Table 1. Ingredients used in each formulation of Italian-Type salami.

\begin{tabular}{lcccc}
\hline Ingredientes & F1 & F2 & F3 & F4 \\
\hline Carne suína (\%) & 86,935 & 86,925 & 86,925 & 86,885 \\
Gordura (toucinho) (\%) & 10,00 & 10,00 & 10,00 & 10,00 \\
Sal (\%) & 2,00 & 2,00 & 2,00 & 2,00 \\
Sal de cura - IBRAC (pó húngaro) (\%) & 0,25 & 0,25 & 0,25 & 0,25 \\
Fixador - IBRAC (eritorbato) (\%) & 0,25 & 0,25 & 0,25 & 0,25 \\
Pimenta branca (\%) & 0,05 & 0,05 & 0,05 & 0,05 \\
Alho em pó (\%) & 0,07 & 0,07 & 0,07 & 0,07 \\
Noz moscada (\%) & 0,02 & 0,02 & 0,02 & 0,02 \\
Realçador de sabor - IBRAC (\%) & 0,10 & 0,10 & 0,10 & 0,10 \\
Açúcar (\%) & 0,30 & 0,30 & 0,30 & 0,30 \\
Cultura Starter - DANISCO (\%) & 0,025 & 0,025 & 0,025 & 0,025 \\
Extrato de própolis pó (\%) & - & - & 0,01 & 0,05 \\
Antioxidante sintético BHT (\%) & - & 0,01 & - & - \\
\hline
\end{tabular}

As análises de perda de peso seguiram a metodologia descrita por MACEDO et al. (2008) e realizadas nos intervalos de 1, 6, 8, 13, 15, 28 e 36 dias após produção, sendo os resultados expressos em porcentagem de perda de peso. Para as análises da cor utilizou-se o sistema CIALAB $\left(L^{*}, a^{*}, b^{*}\right)$ através da leitura em colorímetro (MOD CR-400/ 410 KONICA MINOLTA), iluminante D65 e ângulo de observação de $10^{\circ}$. Com resultados provenientes de $L^{*}, a^{*}$ e $b^{*}$ determinou-se o índice de saturação $\left[C^{*}=\left(a^{* 2}+b^{* 2}\right)^{1 / 2}\right]$ e 0 ângulo de tonalidade $\left[h^{*}=\tan ^{-1}\left(b^{*} / a^{*}\right)\right]$ nos tempos 1,7 e 28 dias durante a maturação e secagem dos salames.

As análises do perfil de textura e força de cisalhamento foram realizadas em Texturômetro (TA. XT. Plus, Texture Analyser-TPA) com os parâmetros de velocidade do pré-teste $(1 \mathrm{~mm} / \mathrm{s})$, teste $(2 \mathrm{~mm} / \mathrm{s})$ e pósteste $(10 \mathrm{~mm} / \mathrm{s})$, força $(5 \mathrm{~N})$; tempo $(1 \mathrm{~s})$ e distância de compressão $(30 \mathrm{~mm})$ previamente ajustados.

Para a força de cisalhamento as amostras foram cortadas com $3 \mathrm{~cm}$ de comprimento, $1 \mathrm{~cm}$ de altura e $1 \mathrm{~cm}$ de largura, utilizando-se a lâmina Warner Bratzler (HDP/BSW) (BOURNE 1978), enquanto que para a determinação do perfil de textura foram calculados os parâmetros de dureza, fraturabilidade, elasticidade, coesividade, adesividade, gomosidade e mastigabilidade.

$\mathrm{O} \mathrm{pH}$ foi analisado com o auxílio de pHmêtro Mettler $340^{\mathrm{TM}}$ conforme metodologia descrita por KINNEBERG \& LINDBERG (2002).

\section{Análises Microbiológicas}

A contagem de coliformes totais e coliformes a $45^{\circ} \mathrm{C}$ foi realizada de acordo com o Official Method of Analysis (KINNEBERG \& LINDBERG 2002). Para a análise de Staphylococcus coagulase positiva e Samonella sp. seguiu-se os procedimentos analíticos da International Standard Organization (ISO 1999) e (ISO 2002), respectivamente.

\section{Análise Sensorial}

A análise sensorial foi realizada por 100 provadores não treinados, conforme instruções contidas no Manual do Instituto Adolfo Lutz (ZENEBON et al. 2008).

As quatro formulações foram codificadas aleatoriamente com três dígitos e avaliadas depois de trinta (30) dias de maturação quanto a aceitação por meio de teste de escala hedônica de nove pontos para os atributos sabor, aroma, cor, textura e aparência global, seguido de teste de intenção de compra utilizando escala hedônica de cinco pontos e teste de preferência mediante ordenação das amostras preferidas, da mais preferida para a menos preferida.

\section{Análise Estatística}

Os resultados obtidos nas análises físicas, $\mathrm{pH}$, teste de aceitação e intenção de compra, foram analisados por meio da análise de variância ANOVA e Teste de Tukey no programa STATISTICA 7.0 (STATSOFT 2005). Os resultados obtidos pelo teste de preferência, foram analisados pelo teste de soma de 
ordens de Friedman (CHAVES 2005).

\section{RESULTADOS E DISCUSSÃO}

\section{Perda de peso e variação da cor}

A perda de peso no decorrer do processo de maturação das quatro formulações do salame tipo italiano é apresentada na Figura 1.

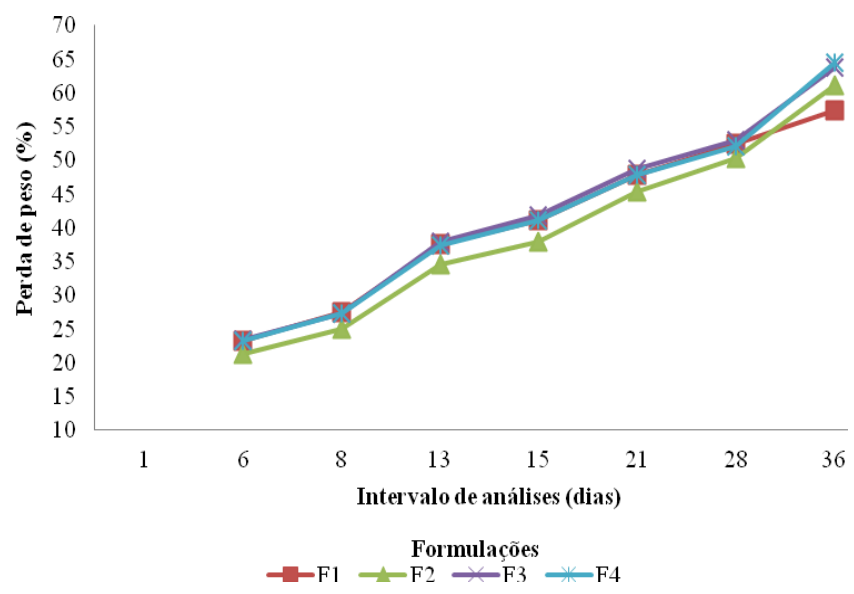

Figura 1. Percentuais médios da perda de peso (\%) no decorrer do período de maturação das diferentes formulações de salame tipo italiano. F1: Formulação 1 (controle); F2: Formulação 2 (0,01\% de BHT); F3: Formulação 3 (0,01\% de própolis); F4: Formulação 4 (0,05\% de própolis).

Figure 1. Mean percentages of weight loss (\%) during the maturation period of the different Italian-Type salami formulations. F1: Formulation 1 (control); F2: Formulation 2 (0.01\% BHT); F3: Formulation 3 (0.01\% propolis); F4: Formulation 4 (0.05\% propolis).

Durante o processo de maturação observou-se aumento no percentual de perda de peso, o que também foi relatado por MARANGONI (2007) em salames tipo italiano formulados com diferentes quantidades de óleo de coentro. As perdas de peso das formulações no $36^{\circ}$ dia de maturação variaram de 57,45 a $64,44 \%$ e até o $26^{\circ}$ dia a amostra F2 (0,01\% de BHT) diferindo estatisticamente das demais, indicando que a incorporação da própolis no salame não afetou o processo de secagem.

Segundo STAHNKE et al. (2001), a aparência de um produto cárneo fermentado é determinada pela cor, que por sua vez é definida pelo brilho, intensidade e tom da cor das partículas cárneas e das partículas de gordura. Os resultados dos índices de cor nos salames estão apresentados na Tabela 2.

A luminosidade $\left(L^{*}\right)$ variou de 37,81 a 58,53 entre as quatro formulações nos três períodos avaliados. Os valores de $L^{*}$ apresentaram um decréscimo ao longo dos 28 dias de maturação com diferença $(p<0,05)$ entre os dias para as formulações F3 e F4. Entre as formulações foi observada diferença para F4 nos tempos 1 e 7 dias enquanto que F3 apresentou diferença somente no $1^{\circ}$ dia. Resultados de $L^{*}$ semelhantes $(37,22$ a 53,64) foram encontrados por CAMPAGNOL (2007) em diferentes tratamentos de salames com cultura starter, CIROLINI et al. (2010) na fabricação de salames com cultura starter nativa $(41,51$ a 59,02$)$ e MACEDO et al. (2008) em salames com culturas lácticas probióticas aos 28 dias de maturação (41,98 a 47,76). De acordo com BERNARDI et al. (2013) a redução da luminosidade no período de maturação é consequência da perda de quantidade de luz refletida, que ocorre com a perda de umidade e concentração dos demais componentes.

Os valores que representam coloração vermelha $\left(a^{*}\right)$ variaram de 10,77 a 15,37 . O aumento dos valores de $a^{*}$ observados nas formulações $\mathrm{F} 1$ e $\mathrm{F} 3$ no $28^{\circ}$ dia de maturação está relacionado com o procedimento de cura e maturação do salame. Nesse processo, o principal pigmento cárneo, a mioglobina, reage com o óxido nítrico que se liga ao ferro heme, formando o composto nitrosomioglobina característico de carnes curadas e é susceptível à oxidação (RUIZ 2011). Valores de $a^{*}$ semelhantes $(11,8$ a 12,7) foram relatados por $\mathrm{HOZ}$ et al. (2004) ao avaliarem amostras de salames.

Os valores de $b^{*}$ variaram de 6,70 a 11,87 , entre as amostras e entre os períodos avaliados. A tonalidade amarela $\left(b^{*}\right)$ obtida para F3 foi diferente $(p<0,05)$ das demais no $1^{\circ}$ dia de maturação, enquanto que $F 2$ diferiu $(p<0,05)$ das outras formulações aos 28 dias. No período avaliado a formulação $F 3$ foi a única que não apresentou alteração nos valores de $b^{*}$. Valores semelhantes de $b^{*}(5,6$ a 8,8$)$ foram relatados por $\mathrm{HOZ}$ et al. (2004) em amostras de salames. 
Tabela 2. Valores médios da luminosidade $\left(L^{*}\right)$, intensidade da cor vermelha $\left(a^{*}\right)$, intensidade da cor amarela $\left(\mathrm{b}^{*}\right)$, índice de saturação $\left(\mathrm{C}^{*}\right)$ e ângulo de tonalidade $\left(\mathrm{h}^{*}\right)$.

Table 2. Mean values of luminosity $\left(L^{*}\right)$, intensity of red color $\left(a^{*}\right)$, intensity of yellow color $\left(b^{*}\right)$, saturation index $\left(C^{*}\right)$ and hue angle $\left(h^{*}\right)$.

\begin{tabular}{|c|c|c|c|c|c|c|}
\hline \multirow[b]{2}{*}{ Dias } & \multirow[b]{2}{*}{ Tratamentos } & \multicolumn{5}{|c|}{ Índices de cor } \\
\hline & & $L^{*}$ & $a^{*}$ & $b^{*}$ & $\mathrm{C}^{*}$ & $\mathrm{~h}^{*}$ \\
\hline \multirow{4}{*}{01} & F1 & $56,40 \pm 0,45^{\text {a } A}$ & $12,22 \pm 0,37^{\mathrm{cB}}$ & $11,87 \pm 0,34^{\text {a } A}$ & $17,79 \pm 0,53^{a b A}$ & $45,00 \pm 2,90^{\text {a } A}$ \\
\hline & $\mathrm{F} 2$ & $58,53 \pm 1,88^{\mathrm{a} A}$ & $15,37 \pm 0,94^{a}$ & $11,35 \pm 0,89^{\underline{a} A}$ & $18,53 \pm 1,25^{\text {a } \mathrm{A}}$ & $36,67 \pm 2,72^{\mathrm{bB}}$ \\
\hline & F3 & $49,34 \pm 1,26^{\mathrm{bA}}$ & $12,30 \pm 0,73^{\mathrm{cA}}$ & $8,65 \pm 0,52^{\mathrm{bA}}$ & $15,44 \pm 0,96^{\mathrm{cA}}$ & $34,65 \pm 1,03^{\mathrm{bA}}$ \\
\hline & $\mathrm{F} 4$ & $49,54 \pm 0,73^{\mathrm{bA}}$ & $13,59 \pm 0,08^{\mathrm{bA}}$ & $11,61 \pm 0,40^{\mathrm{a} A}$ & $16,54 \pm 0,17^{\mathrm{bcA}}$ & $34,28 \pm 1,01^{b A}$ \\
\hline \multirow{4}{*}{07} & F1 & $39,10 \pm 1,27^{b B}$ & $10,77 \pm 0,82^{\mathrm{bC}}$ & $8,02 \pm 0,42^{a \mathrm{a}}$ & $13,55 \pm 0,71^{\mathrm{bc}}$ & $36,40 \pm 2,76^{\mathrm{a} B}$ \\
\hline & $\mathrm{F} 2$ & $42,11 \pm 3,31^{\mathrm{bB}}$ & $12,03 \pm 0,73^{a b B}$ & $8,72 \pm 0,48^{\mathrm{a} B}$ & $15,06 \pm 0,72^{\mathrm{aB}}$ & $35,38 \pm 0,95^{\underline{a} A}$ \\
\hline & F3 & $42,50 \pm 1,21^{\mathrm{bB}}$ & $12,60 \pm 0,88^{a A}$ & $8,40 \pm 0,28^{\mathrm{a} A}$ & $15,22 \pm 0,66^{\mathrm{aA}}$ & $33,25 \pm 1,26^{a \mathrm{a}}$ \\
\hline & $\mathrm{F} 4$ & $47,29 \pm 0,63^{a B}$ & $12,72 \pm 0,47^{\mathrm{aA}}$ & $8,34 \pm 0,71^{\mathrm{aAB}}$ & $15,35 \pm 0,77^{\mathrm{aB}}$ & $33,21 \pm 0,95^{\mathrm{a} A}$ \\
\hline \multirow{4}{*}{28} & F1 & $37,81 \pm 0,97^{\mathrm{a} B}$ & $13,75 \pm 0,61^{\mathrm{aA}}$ & $8,12 \pm 0,65^{\mathrm{aB}}$ & $15,48 \pm 0,68^{\mathrm{a} B}$ & $32,75 \pm 2,46^{\mathrm{a}} \mathrm{B}$ \\
\hline & $\mathrm{F} 2$ & $38,05 \pm 0,57^{\mathrm{aB}}$ & $12,32 \pm 0,71^{\mathrm{bB}}$ & $6,70 \pm 0,34^{\mathrm{bC}}$ & 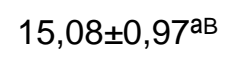 & $29,61 \pm 2,18^{\mathrm{aB}}$ \\
\hline & F3 & $38,19 \pm 1,23^{a c}$ & $13,97 \pm 0,89 \mathrm{aA}$ & $8,53 \pm 0,34^{\mathrm{aA}}$ & $16,55 \pm 0,73^{\mathrm{a} A}$ & $30,82 \pm 3,73^{\mathrm{a} A B}$ \\
\hline & $\mathrm{F} 4$ & $39,83 \pm 0,93^{a c}$ & $13,74 \pm 0,49^{a A}$ & $7,99 \pm 0,29^{a B}$ & $15,65 \pm 0,03^{a A B}$ & $33,64 \pm 5,74^{\underline{a} A}$ \\
\hline
\end{tabular}

Médias seguidas de letras minúsculas iguais na mesma coluna não apresentam diferença significativa $(p>0,05)$ entre as amostras para o mesmo período de tempo pelo Teste Tukey. Médias seguidas de letras maiúsculas iguais na mesma coluna não apresentam diferença significativa $(p>0,05)$ para a mesma amostra nos períodos de 1,7 e 28 dias pelo Teste Tukey. F1: Formulação 1 (controle); F2: Formulação 2 (0,01\% de BHT); F3: Formulação 3 (0,01\% de própolis); F4: Formulação 4 (0,05\% de própolis).

Os valores para os índices de saturação $\left(C^{*}\right)$ variaram de 13,55 a 18,53, sendo que para o período de 28 dias as amostras não diferiram estatisticamente $(p>0,05)$, assim como F3 que não apresentou diferença $(p>0,05)$ para $C^{*}$ nos três períodos avaliados. O ângulo de tonalidade $\left(h^{*}\right)$ apresentou variação de 29,61 a 45 , não tendo sido observada diferenças $(p>0,05)$ entre as quatro formulações nos períodos de 7 e 28 dias. F4 foi a única que não apresentou variação significativa nos valores de $h^{*}(p>0,05)$ nos períodos de avaliação (1, 7 e 28 dias).

\section{Valores de $\mathrm{pH}$}

Os valores de pH durante o período de maturação dos salames são apresentados na Tabela 3.

Tabela 3. Valores de $\mathrm{pH}$ no período de maturação dos salames tipo italiano.

Table 3. $\mathrm{pH}$ values in the maturation period of Italian Type salami.

\begin{tabular}{cccccccc}
\hline Amostras & \multicolumn{7}{c}{ Dias de avaliação de $\mathrm{pH}$} \\
\cline { 2 - 7 } & $1^{\circ}$ dia & $6^{\circ}$ dia & $8^{\circ}$ dia & $13^{\circ}$ dia & $15^{\circ}$ dia & $22^{\circ}$ dia & $28^{\circ}$ dia \\
\hline F1 & $5,61 \pm 0,02^{\underline{a}}$ & $4,69 \pm 0,03^{\underline{a}}$ & $5,10 \pm 0,02^{\mathrm{c}}$ & $5,16 \pm 0,03^{\underline{a}}$ & $5,35 \pm 0,04 b$ & $5,39 \pm 0,02^{\underline{a}}$ & $5,28 \pm 0,03^{\underline{a}}$ \\
F2 & $5,57 \pm 0,03^{\mathrm{b}}$ & $4,71 \pm 0,02^{\underline{a}}$ & $5,14 \pm 0,03^{\mathrm{b}}$ & $5,09 \pm 0,02^{\mathrm{c}}$ & $5,31 \pm 0,03 \mathrm{c}$ & $5,31 \pm 0,02^{\mathrm{b}}$ & $5,29 \pm 0,05^{\underline{a}}$ \\
F3 & $5,58 \pm 0,03^{\mathrm{b}}$ & $4,64 \pm 0,03^{\mathrm{b}}$ & $5,18 \pm 0,04^{\underline{a}}$ & $5,13 \pm 0,02^{\mathrm{b}}$ & $5,41 \pm 0,02^{\underline{a}}$ & $5,37 \pm 0,03^{\underline{a}}$ & $5,20 \pm 0,05^{\mathrm{b}}$ \\
F4 & $5,61 \pm 0,01^{\underline{a}}$ & $4,68 \pm 0,04^{\underline{a}}$ & $5,18 \pm 0,01^{\underline{a}}$ & $5,16 \pm 0,01^{\underline{a}}$ & $5,37 \pm 0,02^{\mathrm{b}}$ & $5,30 \pm 0,05^{\mathrm{b}}$ & $5,22 \pm 0,02^{\mathrm{b}}$
\end{tabular}

Médias seguidas de letras minúsculas iguais na mesma coluna não apresentam diferença significativa $(p>0,05)$ entre as amostras para o mesmo período de tempo pelo Teste Tukey. F1: Formulação 1 (controle); F2: Formulação 2 (0,01\% de BHT); F3: Formulação 3 (0,01\% de própolis); F4: Formulação 4 (0,05\% de própolis).

Os valores de $\mathrm{pH}$ demonstraram diferenças $(\mathrm{p}<0,05)$ significativas entre algumas amostras, bem como em relação ao tempo de maturação. Todas as amostras apresentaram queda considerável do pH no 6을 dia de maturação em relação ao $1^{\circ}$ dia, o que tem relação com a fermentação do produto em função do metabolismo das bactérias ácido lácteas presentes na cultura starter adicionada, já que acidifica o meio durante esse processo. 
Por outro lado, com o passar dos dias notou-se um aumento de $\mathrm{pH}$ para todas as formulações. De acordo com CACCIOPPOLI et al. (2006), tal comportamento pode ocorrer devido à descarboxilação e desaminação dos aminoácidos que liberam amônia, que por ser um tipo de sal deixa o meio mais alcalino, embora ainda ocorra acidificação no produto, mesmo que mais atenuada já que a quantidade de água livre está mais reduzida ao fim do processo de fermentação e desidratação, reduzindo assim o metabolismo das bactérias ácido lácteas.

SCHEID et al. (2003) e MARANGONI \& MOURA (2011) observaram comportamento de pH semelhante aos encontrados nesse estudo durante a maturação de salames tipo italiano adicionados de cravo-da-índia e óleo essencial de coentro, respectivamente.

Uma análise do que foi relatado por KOMIYAMA et al. (2010) permite considerar que os valores de $\mathrm{pH}$ encontrados nas formulações $\mathrm{F} 3$ e F4 aos 28 dias de maturação podem ter relação com os valores obtidos para a força de cisalhamento e dureza dessas formulações. Isso porque, segundo os autores supracitados, quanto menor o pH de um produto cárneo, mais intensa tende a ser a desnaturação protéica em função da proximidade do ponto isoelétrico da maior parte das proteínas miofibrilares $(5,2-5,3)$, logo, implicando em maior proteólise e consequentemente, maior maciez do produto.

De acordo com TERRA et al. (2004) os valores de pH nos produtos cárneos são essenciais para a formação das características sensoriais, uma vez que facilita as enzimas tissulares e microbianas a degradarem os lipídios e proteínas precursoras do flavor. Segundo TERRA (1997), valores de pH mais baixos também mantém a estabilidade microbiológica, sendo ainda, responsável pela liberação de água do produto fermentado devido a troca do estado sólido para o estado de gel das proteínas musculares conferindo textura característica do produto, desidratando o mesmo e reduzindo o seu tempo de maturação.

\section{Força de cisalhamento e perfil de textura}

Os resultados obtidos para a força de cisalhamento e o perfil de textura para as quatro formulações de salames adicionados de própolis podem ser observados na Tabela 4.

Tabela 4. Valores médios para força e perfil de textura (TPA), do Salame Tipo Italiano aos 34 dias de maturação.

Table 4. Mean values to force and texture profile (TPA) of Italian-Type salami at 34 days of maturation.

\begin{tabular}{|c|c|c|c|c|}
\hline \multirow{2}{*}{$\begin{array}{c}\text { Parâmetros - Período de } \\
\text { maturação }\end{array}$} & \multicolumn{4}{|c|}{ Formulações } \\
\hline & F1 & F2 & F3 & F4 \\
\hline Força $(\mathrm{N})-6^{\circ}$ dia & $14,12 \pm 0,99 \mathrm{aB}^{*}$ & $7,64 \pm 0,98^{\mathrm{cB}}$ & $12,37 \pm 0,86^{\mathrm{ab}^{*}}$ & $11,57 \pm 0,59^{\mathrm{bB}}{ }^{*}$ \\
\hline Força $(\mathrm{N})-28^{\circ}$ dia & $18,57 \pm 1,16^{c A^{*}}$ & $28,15 \pm 1,90^{a A^{\star}}$ & $21,11 \pm 2,12^{\mathrm{bA}^{*}}$ & $14,84 \pm 1,82^{\mathrm{d} A^{*}}$ \\
\hline Dureza $(\mathrm{N})-34^{\circ} \mathrm{dia}$ & $264,04 \pm 6,73^{c}$ & $443,35 \pm 6,62^{\mathrm{a}}$ & $346,94 \pm 14,71^{b}$ & $247,89 \pm 17,31^{\mathrm{c}}$ \\
\hline Coesividade $-34^{\circ}$ dia & $0,44 \pm 0,05^{\mathrm{a}}$ & $0,27 \pm 0,05^{b}$ & $0,43 \pm 0,04^{a}$ & $0,43 \pm 0,09^{a}$ \\
\hline Fraturabilidade(\%) - $34^{\circ} \mathrm{dia}$ & $14,99 \pm 1,45^{\mathrm{ab}}$ & $9,45 \pm 1,03^{c}$ & $16,39 \pm 1,58^{\mathrm{a}}$ & $12,78 \pm 0,42^{b}$ \\
\hline Mastigabilidade $-34^{\circ}$ dia & $63,09 \pm 3,02^{b}$ & $65,09 \pm 10,80^{\mathrm{b}}$ & $87,20 \pm 9,89 a$ & $66,20 \pm 4,57^{b}$ \\
\hline Gomosidade $-34^{\circ}$ dia & $107,27 \pm 6,73^{b}$ & $82,20 \pm 7,58^{c}$ & $158,55 \pm 14,94^{\underline{a}}$ & $104,41 \pm 4,00^{\mathrm{bc}}$ \\
\hline Adesividade (N.s) $-34^{\circ}$ dia & $-0,018 \pm 0,01^{a}$ & $-0,025 \pm 0,01^{a}$ & $-0,058 \pm 0,04^{a}$ & $-0,025 \pm 0,01^{a}$ \\
\hline Elasticidade (\%) - $34^{\circ}$ dia & $64,35 \pm 0,23^{a}$ & $63,02 \pm 0,99 \mathrm{a}$ & $63,86 \pm 2,85^{\mathrm{a}}$ & $62,04 \pm 0,93^{\underline{a}}$ \\
\hline
\end{tabular}

Médias seguidas de letras minúsculas iguais na mesma linha não apresentam diferença significativa $(p>0,05)$ pelo Teste Tukey entre as diferentes amostras. *Médias seguidas de letras maiúsculas na mesma linha não apresentam diferença significativa $(p>0,05)$ quanto a força de cisalhamento para cada amostra entre $\circ 6^{\circ}$ e $\circ 28^{\circ}$ dia de maturação pelo Teste Tukey. F1: Formulação 1 (controle); F2: Formulação 2 (0,01\% de BHT); F3: Formulação 3 (0,01\% de própolis); F4: Formulação 4 (0,05\% de própolis).

No sexto dia de maturação a formulação F2 apresentou força de cisalhamento inferior $(p<0,05)$ em relação às demais, porém, aos 28 dias o resultado inverteu tendo a mesma diferindo significativamente das outras com maior força de cisalhamento. Infere-se, portanto, que a adição de BHT como antioxidante pode ter levado a uma redução de umidade mais branda nos primeiros dias de fermentação, tendo uma aceleração no processo de desidratação com o passar do tempo.

Para TERRA et al. (2007), esse comportamento pode proporcionar ao produto menor estabilidade microbiológica nos primeiros dias de fermentação, visto (GALÁN et al. 2011) a existência de água em quantidade suficiente para o desenvolvimento de microorganismos deteriorantes, além do produto não se encontrar no estágio de fermentação adequado para o abaixamento suficiente do $\mathrm{pH}$, o que se 
caracterizaria outra barreira antimicrobiana juntamente com a baixa umidade.

Foi observado um aumento da força de cisalhamento aos 28 dias de maturação em comparação aos resultados obtidos nas análises realizadas no $6^{\circ}$ dia de fermentação para todas as amostras. Essa observação pode ter relação com a perda de umidade, visto sua aceleração ao longo da maturação. Entretanto, uma queda repentina de umidade pode interferir negativamente na textura do salame, implicando em maior proteólise e consequentemente, maior maciez do produto final, característica essa não desejada para o salame tipo italiano (TERRA et al. 2005).

LIMA (2009) elaborou salames com carne de cordeiro e obteve força de cisalhamento entre 3,56 kgf e $5,39 \mathrm{kgf}$ no $22^{\circ}$ dia de maturação, resultados esses superiores aos encontrados nesse estudo para os salames com 28 dias de maturação, os quais convertidos para kgf apresentaram força de cisalhamento entre $1,5 \mathrm{kgf}$ e $2,8 \mathrm{kgf}$ se apresentando, portanto, menos rígidos mesmo que com seis dias a mais de fermentação em relação aos salames analisados pelo autor supracitado. A divergência entre os valores obtidos nesse estudo e aqueles encontrados por LIMA (2009) pode ser justificada pela utilização de carnes de diferentes espécies.

Segundo GRANADOS et al. (2013), a textura de um alimento pode ser avaliada de forma instrumental ou por meio de testes sensoriais com provadores. Para a análise instrumental do perfil de textura algumas propriedades devem ser consideradas em um alimento, tais como dureza, adesividade, fraturabilidade, coesividade, elasticidade, gomosidade e mastigabilidade (HLEAP \& VELASCO 2010).

A dureza é definida como a força requerida para a compressão do alimento pelos dentes e está diretamente relacionada com as propriedades de coesividade e fraturabilidade, ou seja, quanto mais duro o alimento, menor tende a ser a força necessária de compressão para que ocorra o rompimento do alimento (coesividade) e menor a força exigida para que o alimento se quebre (fraturabilidade) (HERRERO et al. 2008).

Proporcionalmente, existe uma tendência da amostra com resultados superiores de dureza e inferiores para coesividade e fraturabilidade apresentar menores resultados para mastigabilidade, gomosidade, adesividade e elasticidade (LORENZO \& FRANCO 2012), o que se torna um problema quando se trata da formulação de salame tipo italiano, visto ser sensorialmente desejável que o produto apresente uma textura rígida (MAPA 2000), porém, que não seja quebradiço.

Esse comportamento foi percebido na formulação F2, adicionada de BHT, porém sem diferença entre as demais formulações com relação a adesividade e elasticidade. Seguindo essa linha de raciocínio, uma textura dura e valores intermediários para coesividade, fraturabilidade e gomosidade seriam mais interessantes do ponto de vista sensorial do que o observado para F2 contendo antioxidante sintético. Logo, as formulações controle (F1) e F4, adicionada de $0,05 \%$ de própolis (antioxidante natural) podem ser tidas como aquelas com melhores resultados para o perfil de textura, uma vez que não foram diferentes significativamente $(p>0,05)$ entre si e apresentaram um melhor comportamento mecânico entre as amostras avaliadas.

BERNARDI et al. (2013) explicaram que a utilização de antioxidantes sintéticos ou naturais em produtos cárneos maturados é extremamente necessária, pois, devido à desidratação dos mesmos as gorduras são concentradas e por isso uma maior probabilidade de desencadear o processo de oxidação lipídica nesses alimentos, e consequentemente, a redução do seu prazo de validade.

FIEIRA et al. (2015) encontraram maiores valores de dureza $(13,79$ a 31,75$)$ e mastigabilidade $(1,65$ a $4,20)$ em salames tipo italiano com redução de sódio. Provavelmente, os valores mais elevados para dureza $(247,89$ a 443,35$)$ e mastigabilidade $(63,09$ a 87,20$)$ obtidos nesse estudo em relação aos observados pelos autores mencionados estejam relacionados à redução na quantidade de cloreto de sódio. De acordo com HORITA et al. (2011), o $\mathrm{NaCl}$ normalmente adicionado aos produtos cárneos produzem a força iônica necessária para a dissolução e extração das proteínas miofibrilares, que por sua vez são responsáveis pela emulsificação, gelatinização e capacidade de retenção de água. Logo, quando a concentração de $\mathrm{NaCl}$ foi reduzida, a quantidade de proteína extraída foi diminuída alterando as propriedades da textura do produto desenvolvido por FIEIRA et al. (2015) e, consequentemente, o deixando mais macio.

\section{Análise Sensorial}

Previamente à realização da análise sensorial as amostras foram submetidas a avaliação microbiológica visando garantir a segurança dos provadores. Todas as formulações desenvolvidas encontraram-se dentro dos padrões microbiológicos exigidos pela legislação (ANVISA 2001).

Os valores médios obtidos na avaliação sensorial das quatro formulações de salames adicionados de extrato de própolis mediante teste de aceitação, intenção de compra e teste de preferência encontram-se apresentados na Tabela 5. 
Tabela 5. Pontuação média dos atributos aparência global, aroma, cor, textura, sabor e teste de intenção de compra, avaliados nas formulações de Salame tipo italiano.

Table 5. Average score of the attributes global appearance, aroma, color, texture, flavor and intention of purchase test, evaluated in Salami Italian-Type formulations.

\begin{tabular}{|c|c|c|c|c|c|c|}
\hline \multirow[b]{2}{*}{ Formulações } & \multicolumn{6}{|c|}{ Atributos } \\
\hline & Sabor & Aroma & Cor & Textura & $\begin{array}{l}\text { Aparência } \\
\text { global }\end{array}$ & $\begin{array}{c}\text { Intenção de } \\
\text { compra }\end{array}$ \\
\hline $\mathrm{F} 1$ & $7,58 \pm 1,72^{a}$ & $7,76 \pm 1,42^{a}$ & $7,79 \pm 1,26^{a}$ & $7,55 \pm 1,47^{a}$ & $7,75 \pm 1,31^{a}$ & $4,04 \pm 1,25^{a}$ \\
\hline $\mathrm{F} 2$ & $7,82 \pm 1,26$ a & $7,79 \pm 1,20$ a & $7,80 \pm 1,35$ a & $7,46 \pm 1,40$ a & $7,87 \pm 1,16$ a & $4,12 \pm 1,06 \mathrm{a}$ \\
\hline F3 & $7,35 \pm 1,52 \mathrm{a}$ & $7,70 \pm 1,05$ a & $7,75 \pm 1,13^{a}$ & $7,51 \pm 1,24$ a & $7,71 \pm 1,13^{a}$ & $3,81 \pm 1,10 \mathrm{ab}$ \\
\hline F4 & $7,64 \pm 1,45^{a}$ & $7,36 \pm 1,39$ a & $7,17 \pm 1,68 \mathrm{~b}$ & $7,40 \pm 1,46$ a & $7,73 \pm 1,27^{a}$ & $3,54 \pm 1,30^{b}$ \\
\hline
\end{tabular}

Médias seguidas de letras iguais na mesma coluna não apresentam diferença significativa $(p>0,05)$ pelo Teste Tukey. F1: Formulação 1 (controle); F2: Formulação 2 (0,01\% de BHT); F3: Formulação 3 (0,01\% de própolis); F4: Formulação 4 (0,05\% de própolis).

As diferentes formulações de salame tipo italiano não diferiram $(p>0,05)$ entre si no teste de aceitação com nível de $95 \%$ de confiança para aparência global, aroma, textura e sabor, tendo a amostra F4 diferido $(p<0,05)$ das demais com relação a cor, com a menor pontuação média. $F 1$, F2, e F3 apresentaram maior nota média $(p<0,05)$ para o atributo cor, embora F2 tenha apresentado menor valor na avaliação instrumental aos 28 dias de maturação para o índice que representa a coloração vermelha, desejada em produtos cárneos (TERRA et al. 2005). Essa observação permite dizer que os resultados da análise instrumental de cor divergiram dos observados na análise sensorial para essa formulação (F2), especificamente.

BACKES et al. (2013) avaliaram diferentes tratamentos de salame tipo italiano com substituição parcial da gordura suína por emulsão de óleo de canola e não observaram diferença $(p>0,05)$ de aceitação entre os tratamentos durante os testes sensoriais realizados. DALLA SANTA (2008), assim como BACKES et al. (2013) também não verificaram diferença $(p>0,05)$ entre as amostras de salame desenvolvidas para os atributos sabor, aroma, cor, textura e aparência visual.

No presente estudo os julgadores demonstraram maior intenção de compra $(p<0,05)$ para as formulações controle (F1), F2 e F3 em relação a F4, embora F3 e F4 não terem diferido $(p>0,05)$ entre si. Apesar de $\mathrm{F} 4$ ter se apresentado significativamente igual $(p>0,05)$ às demais formulações com relação à maioria dos atributos sensoriais analisados, foi a menos preferida pelo teste de preferência por ordenação, enquanto que F2 foi a mais preferida entre todas as amostras avaliadas, seguida de F1 e F3.

Os resultados dos testes sensoriais demonstram que embora F4 tenha apresentado menor nota média para cor $(7,17)$ no teste de aceitação, isso não interferiu na sua aparência global, porém, pode ter levado a menor intenção de compra em relação a F1 e F2, bem como menor preferência em comparação a F1 e F2. Isso porque, segundo BACKES et al. (2013) a cor é considerada um importante atributo de qualidade da carne e seus derivados, com influência considerada na sua aceitação por parte dos consumidores.

Observou-se nesse estudo que apesar de F4 ter apresentado resultados interessantes para o perfil de textura na análise instrumental, a mesma não se comportou entre as melhores aceitas durante os testes sensoriais realizados por julgadores.

As Formulações F1, F2, F3 e F4 obtiveram percentuais de certeza de compra na ordem de $80,8 \%$, $82,4 \%, 76,2 \%$ e $70,8 \%$, respectivamente. Segundo o que informa o manual do Instituto Adolfo Lutz (ZENEBON et al. 2008), o grau de certeza de compra entre as amostras deve ser maior ou igual a 70\%, o que indica, portanto, uma boa aceitação dos produtos desenvolvidos e testados.

\section{CONCLUSÃO}

A adição de $0,05 \%$ de própolis em substituição ao BHT proporcionou resultados interessantes quanto ao perfil de textura para o salame tipo italiano, com características físicas esperadas para esse produto cárneo. O grau de certeza de compra por parte dos julgadores mostrou que todas as formulações testadas podem receber boa aceitação pelos consumidores em caso de serem disponibilizadas no mercado e que, consequentemente, a adição do extrato de própolis em substituição ao antioxidante sintético não contribuiu para a rejeição do produto. 


\section{AGRADECIMENTOS}

Os autores agradecem a Universidade Tecnológica Federal do Paraná.

\section{REFERÊNCIAS}

ALCANTARA M et al. 2012. Principais microorganismos envolvidos na deterioração das características sensoriais de derivados cárneos. Revista Brasileira de Higiene e Sanidade Animal 6: 1-20.

ALVES E \& KUBOTA EH. 2013. Conteúdo de fenólicos, flavonoides totais e atividade antioxidante de amostras de própolis comerciais. Revista de Ciências Farmacêuticas Básica e Aplicada 34: 37-41.

ANVISA. 2001. Agência Nacional de Vigilância Sanitária. Resolução RDC n. 12, de 02 de janeiro de 2001. Regulamento Técnico sobre padrões microbiológicos para alimentos. DOU 10/01/2001. Seção 1.

BACKES AM et al. 2013. Características físico-químicas e aceitação sensorial de salame tipo italiano com adição de óleo de canola. Semina: Ciências Agrárias 34: 3709-3720.

BELEDELLI BD et al. 2011. Avaliação de salame tipo italiano adicionado de selênio. Perspectiva 35: 57-67.

BERNARDI S et al. 2013. Italian-type salami with propolis as antioxidant. Italian Journal of Food Sciences 25: $433-441$.

BOURNE MC. 1978. Texture profile analysis. Food Technology 32: 62-66.

BRUSCHI ML. 2006. Desenvolvimento e caracterização de sistemas de liberação de própolis intrabolsa periodontal.

Tese (Doutorado em Ciências Farmacêuticas). Ribeirão Preto: USP. 320p.

CACCIOPPOLI $\mathrm{J}$ et al. 2006. Aminas bioativas e características físico-químicas de salames tipo italiano. Arquivo Brasileiro de Medicina Veterinária e Zootecnia 58: 648-657.

CAMPAGNOL PCB. 2007. Cultura de starter produzida em meio de cultura de plasma suíno e antioxidante natural na elaboração do salame. Dissertação (Mestrado em Ciência e Tecnologia de Alimentos). Santa Maria: UFSM. 74p.

CHAVES JBP. 2005. Métodos de diferença em avaliação sensorial de alimentos e bebidas. 3.ed. Viçosa: Editora UFV. $91 \mathrm{p}$.

CIROLINI A et al. 2010. Salame Tipo Italiano elaborado com culturas starters nativas. Ciências e Tecnologia de Alimentos 30: 171-179.

DALLA SANTA OR. 2008. Avaliação da qualidade de salames artesanais e seleção de Culturas starter para produção de salame tipo italiano. Tese (Doutorado em Tecnologia de Alimentos). Curitiba: UFPR. 174p.

FIEIRA C et al. 2015. Partial replacement of sodium chloride in Italian salami and the influence on the sensory properties and texture. Acta Scientiarum 37: 293-299.

GALÁN I et al. 2011. Effects of ionising irradiation on quality and sensory attributes of ready-to-eat dry fermented sausages enriched with folic acid. International Journal of Food Science and Technology 46: 469-477.

GRANADOS $\mathrm{C}$ et al. 2013. Análisis proximal, sensorial y de textura de salchichas elaboradas con subproductos de la industria procesadora de atún (Scombridae thunnus). Información Tecnológica 24: 29-34.

HERRERO AM et al. 2008. Tensile properties of cooked meat sausages and their correlation with texture profile analysis (TPA) parameters and physico-chemical characteristics. Meat Science 80: 690-696.

HLEAP JI \& VELASCO AV. 2010. Análisis de las propiedades de textura durante el almacenamiento de salchichas elaboradas a partir de Tilapia roja (Oreochromis sp.). Revista de la Facultad de Ciencias Agropecuarias 8: 46-56.

HORITA CN et al. 2011. Physico-chemical and sensory properties of reduced-fat mortadella prepared with blends of calcium, magnesium and potassium chloride as partial substitutes for sodium chloride. Meat Science 89: 426-433.

HOZ L et al. 2004. Development of an n-3 fatty acid and a-tocopherol enriched dry fermented sausage. Meat Science 67: 485-495.

ISO. 1999. International Standard Organization. Microbiology of food and animal feeding stuffs. Horizontal method for the enumeration of coagulase-positive staphylococci (Staphylococcus aureus and other species) - Part 1: Technique using Baird. International Standard Organization. ISO 6888-1.

ISO. 2002. International Standard Organization. Microbiology of food and animal feeding stuffs. Horizontal method for the detection of Salmonella spp. 4.ed. ISO 6579.

KINNEBERG KM \& LINDBERG KG. 2002. Dry rehydratable film method for rapid enumeration of coliforms in foods (3M Petrifilm Rapid Coliform Count plate): collaborative study. Journal of Official Method of Analysis 85: 56-71.

KOMIYAMA ICM et al. 2010. Qualidade físico-química e sensorial da carne de peito de matrizes pesadas de descarte. Ciência Rural 40: 1623-1629.

LIMA IA. 2009. Elaboração e caracterização de Salame de Cordeiro Santa Inês. Dissertação (Mestrado em Engenharia de Alimentos). Itapetinga: UESB. 76p.

LORENZO JM \& FRANCO D. 2012. Fat effect on physico-chemical, microbial and textural changes through the manufactured of dry-cured foal sausage lipolysis, proteolysis and sensory properties. Meat Science 92: 704-714.

MACEDO REF et al. 2008. Desenvolvimento de embutido fermentado por Lactobacillus probióticos: características de qualidade. Ciência e Tecnologia de Alimentos 28: 509-519.

MAPA. 2000. Ministério da Agricultura, Pecuária e Abastecimento. Instrução Normativa n. 22, de 31 de julho de 2000. Regulamento técnico de identidade e qualidade do salame tipo italiano. DOU 03/08/2000. Seção 1.

MAPA. 2001. Ministério da Agricultura, Pecuária e Abastecimento. Secretaria de Defesa Agropecuária. Instrução Normativa n. 3, de 19 de Janeiro de 2001. Regulamento técnico para fixação de identidade e qualidade de própolis. Anexo VI.

MARANGONI C. 2007. Atividade antioxidante do óleo essencial de coentro (Coriandrum sativum L.) em salame italiano. 
Dissertação (Mestrado em Ciências Ambientais). Chapecó: Unochapecó. 132p.

MARANGONI C \& MOURA NF. 2011. Antioxid antactivity of essential oil from Coriandrum sativum L. in Italian salami. Ciência e Tecnologia de Alimentos 31: 124-128.

MATHIAS SP et al. 2010. Alterações oxidativas (cor e lipídios) em presunto de peru tratado por alta pressão hidrostática (APH). Ciência e Tecnologia de Alimentos 30: 852-857.

OLIVEIRA DF et al. 2013. Alternativas para um produto cárneo mais saudável: uma revisão. Brazilian Journal Food Technology 16: 163-174.

OLIVO R. 2006. Alterações Oxidativas em Produtos Cárneos. In: SHIMOKOMAKI M et al. (Ed.). Atualidades em Ciência e Tecnologia de Carnes. São Paulo: Livraria Varela. p.155-162.

PEREIRA ALF et al. 2010. Estabilidade oxidativa de mortadelas contendo extrato da casca da manga (Mangifera indica L.). Brazilian Journal of Food Technology 13: 293-298.

RUIZ JN. 2011. Aplicação de microorganismo probióticos nas formas livre e microencapsulada em salame tipo italiano. Dissertação (Mestrado em Ciência e Tecnologia de Alimentos). Piracicaba: ESALQ. 123p.

SCHEID GA et al. 2003. Avaliação físico-química e sensorial de salame tipo italiano contendo diferentes concentrações de cravo-da-índia (Eugenia caryophyllus). Ciência Agrotécnica. Edição Especial: 1576-1583.

STAHNKE LH et al. 2001. Maturity accelerations by Staphylococcus carnosus in fermented sausage - relationship between maturity and flavor compounds. Journal of Food Science 67: 1914-1921.

STATSOFT Inc. 2005. Statistica. Data Analysis Software System, Version 7.1. http://www.statsoft.com

TERRA NN. 1997. Fermentação como fator de segurança e qualidade para o consumidor. Revista Nacional da Carne 239: 26-32.

TERRA ABM et al. 2004. Particularidades na fabricação de salame. São Paulo: Livraria Varela. 152p.

TERRA NN. 2005. Apontamentos sobre tecnologia de carnes. São Leopoldo: Editora Unisinos. 216p.

TERRA NN et al. 2007. Water activity, $\mathrm{pH}$, moisture and growth of Staphylococcus xylosus during processing and storage of cured, matured and fermented pork shoulder. Ciência e Tecnologia de Alimentos 27: 756-760.

ZANARDI E et al. 2002. Lipid and colour stability of Mylano-type sausages: effect of packing conditions. Meat Science 61: 7-14.

ZENEBON O et al. 2008. Métodos físico-químicos para análise de alimentos. 4.ed. São Paulo: Instituto Adolfo Lutz. 1020p. 